\title{
Ichthyosis uteri complicated by poorly differentiated endometrial adenocarcinoma with squamous differentiation
}

\author{
Haiyan Shi, Xiaoduan Chen, Suojiang Zhang, Caiyun Zhou \\ Department of Pathology, the Affiliated Women's Hospital of Zhejiang University School of Medicine, Hangzhou 310003, PR China \\ Przegląd Menopauzalny 2013; 6: 449-452
}

\section{Summary}

Endometrial squamous metaplasia is a common type of metaplasia. Wide endometrial squamous metaplasia, or ichthyosis uteri, is a rare form of the disease. Malignant transformation based on ichthyosis uteri alone or ichthyosis uteri complicated by other malignant lesions is even rarer. In this study, we report a case of ichthyosis uteri complicated by poorly differentiated endometrial adenocarcinoma. A 74-year-old patient visited the doctor's office after suffering from irregular and small amounts of vaginal hemorrhage for half a month. Gynecological examination showed a 2-month pregnancy-like uterine enlargement. Histopathological examination confirmed two types of lesions: poorly differentiated endometrioid adenocarcinoma and wide replacement of a large area of endometrium by mature squamous epithelium. Based on previous studies and the current report, the malignant potential of ichthyosis uteri or its simultaneous occurrence with other malignant lesions cannot be completely excluded, although the lesion observed was only considered a benign lesion.

Key words: endometrial metaplasia, endometrial carcinoma, ichthyosis uteri.

\section{Introduction}

Endometrial squamous metaplasia is a common type of metaplasia that may occur either in multiple benign endometrial lesions, such as endometrial hyperplasia, endometritis, and endometrial polyps, as well as in endometrial adenocarcinoma. Endometrial squamous metaplasia has two forms, the common case of mulberry-like squamous metaplasia and the rare case that involves the replacement of endometrial surface by stratified squamous epithelium. When the endometrial surface is widely replaced by squamous epithelium, an even rarer endometrial pathological condition occurs, which is referred to as ichthyosis uteri. Ichthyosis uteri was first proposed by Zeller in 1885, who found that wide endometrial squamous metaplasia was caused by caustic (such as formalin and iodine) infusion into the uterine cavity or similar lesions, such as pyometra, endometrial tuberculosis, endometrial hyperplasia, and so on [1]. Ichthyosis uteri has long been believed to be subject to benign lesions in pathology, although it is often clinically misdiagnosed as carcinoma. However, some studies have reported that ichthyosis uteri is accompanied by squamous epithelial dysplasia or even squamous cell carcinoma; thus, these findings pose doubts about the neoplastic potential [2-9].
In the current study, we report a rare case of ichthyosis uteri complicated by poorly differentiated endometrial adenocarcinoma. We also discuss the cause and biological behaviors of ichthyosis uteri based on previous studies.

\section{Case report}

A 74-year-old patient visited the doctor's office after suffering from irregular and small amounts of vaginal hemorrhage for half a month. Gynecological examination showed a 2-month pregnancy-like uterine enlargement. B ultrasound scanning showed heterogeneous echoes of a $5.6 \mathrm{~cm} \times 5.9 \mathrm{~cm} \times 4.0 \mathrm{~cm}$ mass in the uterine cavity. Based on fractional curettage pathological reports, the curetted mass from the uterine cavity was believed to be a malignant tumor, which was first considered as poorly differentiated adenocarcinoma, and the curetted masses from the neck tube were small amounts of endometrium and keratinizing squamous epithelium. The patient underwent total hysterectomy and bilateral salpingo-oophorectomy. The operation was accomplished smoothly. The patient had no history of tuberculosis or endometritis. She received tubal ligation 46 years before

Address for correspondence:

Haiyan Shi, Department of Pathology, The Affiliated Women's Hospital of Zhejiang University School of Medicine, Hangzhou 310003, PR China,

tel. 86-571-8706501-1702, fax: 86-571-87061878, e-mail: haiyanshicn@163.com 
without a history of receiving an intrauterine device or hormone replacement therapy.

\section{Macro examination}

The volume of the uterus was $11 \mathrm{~cm} \times 8 \mathrm{~cm} \times 3 \mathrm{~cm}$ with an abnormal shape and a slick serous membrane. After the uterus was cut open, the cavity was found to be filled up with dated blood, and an ulcer type of mass (4 cm in diameter) was observed in the posterior wall located near the uterine bottom. The mass had infiltrated into the full thickness of the uterine wall until it reached the serous membrane. The endometrium around the mass was gray with tiny papillae that densely covered the surface. A myoma $(3 \mathrm{~cm}$ in diameter) was observed between the bottom uterine muscle layers. The cervical mucous membrane was smooth and no obvious mass was observed on its incisal surface. Bilateral ovaries and fallopian tubes exhibited atrophy and post-ligation changes (Fig. 1).

\section{Microscopic examination}

The tumor was poorly differentiated with cells distributed in closely packed nests and solid sheets (Fig. 2). Gland-like structure formation trends were observed in a few regions (Fig. 3). In the cell nests, distinct squamous differentiation regions could be observed (Fig. 4). The tumor primarily grew endophytically, infiltrating into the full thickness of the muscle layers until it reached the serous layer. No involvement of the cervix or the regions out of the uterus was observed. The tumor was pathologically determined to be at Stage IB according to the International Federation of Gynecology and Obstetrics staging standards (2009).

The tumor surface and the endometrium around the tumor were widely covered with stratified squamous epithelia (Fig. 2); the latter indicated noticeable hyperplasia, as well as papilloma-like growth in some regions. However, the cell differentiated maturely without heteromorphisms and karyokinesis was not active. No evidence of human papillomavirus (HPV) infection such as koilocytosis was detected. A small amount of endometrial glands were found under the squamous epithelium, which showed atrophic changes and were surrounded by numerous infiltrating lymphocytes and plasmacytes (Fig. 5).

\section{Immunohistochemistry}

Tumor cells showed diffused cytokeratin (CK) positivity. High and low molecular CK and protein 63 (P63) were partially positive, where the cells positive with high molecular CK and P63 were concentrated in the squamous differentiation regions. Neuroendocrine markers, such as neuron-specific enolase, synaptophysin, and chromogranin A, as well as estrogen receptor, progesterone receptor, and P16, were negative, whereas P53 was widely and strongly positive. P16 was negative in the endometrial squamous epithelial cells, and the cells with positive ki67 were dispersed in the basal layer.

\section{Discussion}

The uterine tumor in the present study revealed that the tumor cells were distributed in a nest-like fashion, with diffused positive CK and negative neuroendocrine markers, which indicated that the uterine tumor was poorly differentiated carcinoma. In the tumor, a large area of squamous differentiation could be observed; thus, a differential diagnosis between poorly differentiated adenocarcinoma that was complicated by squamous differentiation and squamous cell carcinoma was needed. Endometrial squamous cell carcinoma is a clinically rare disease and has only been reported in only over 70 patients. Among these reported cases, the most common condition is manifested by the diffusion or metastasis of cervical squamous carcinoma into the uterine cavity. Therefore, in the present case, the materials were fully drawn from the cervix. Neither squamous carcinoma or intraepithelial neoplasm nor positive HPV or P16 expression was detected, which excluded the diffusion of cervical squamous carcinoma toward the endomembrane. In addition, the diagnosis of primary squamous cell carcinoma of endometrium must consider the exclusion of a more common clinical condition, that is, endometrioid adenocarcinoma with predominantly squamous differentiation, as a premise. In the present

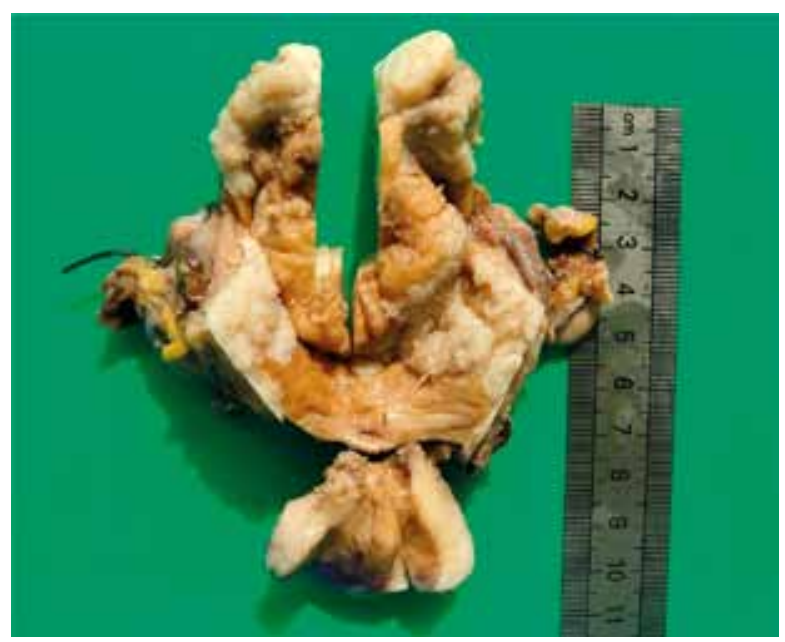

Fig. 1. Macro observation of the sample. An ulcer type of mass with a diameter of $4 \mathrm{~cm}$ can be observed in the posterior uterine wall close to the uterine bottom, and the endometrium around the mass shows gray color with tiny papillae densely covering the surface 


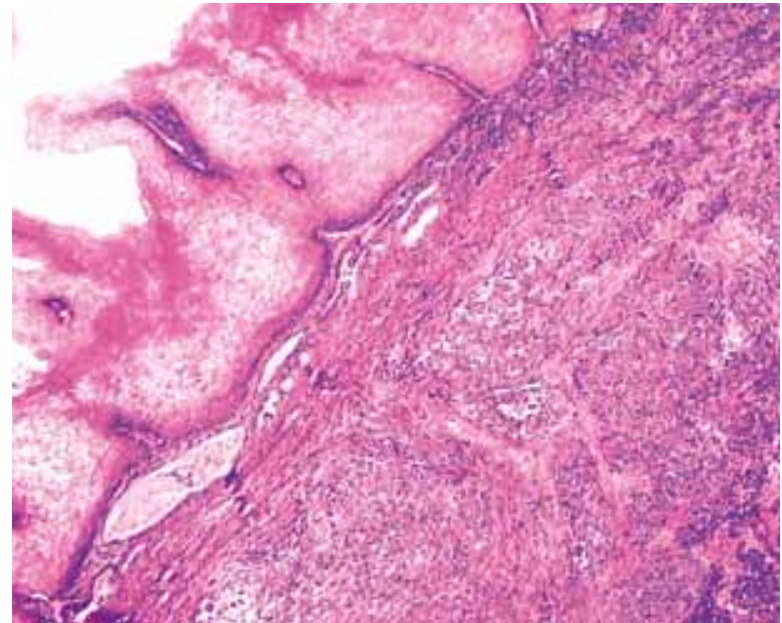

Fig. 2. Microscopic observation of the sample. Tumor cells are distributed in closely packed nests and solid sheets, and the tumor is widely covered by matured stratified squamous epithelium. (HE; 100x)

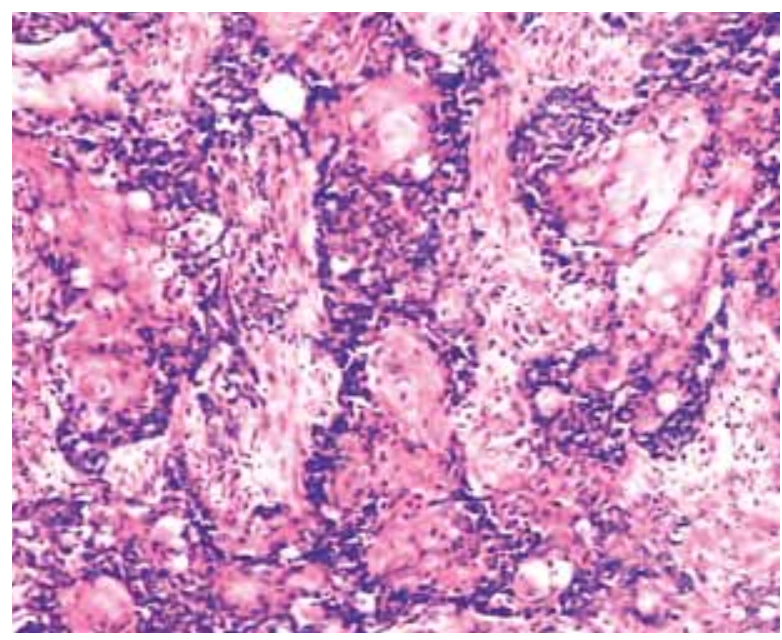

Fig. 4. Noticeable squamous differentiation regions in the tumor nests (HE; 200x)

case, gland-like structures could be observed in a few tumor regions, which further confirmed the diagnosis of poorly differentiated endometrioid adenocarcinoma complicated by squamous differentiation.

Histopathological examination in the present case confirmed two types of lesions. One was poorly differentiated endometrioid adenocarcinoma and the other was ichthyosis uteri, which was manifested by the wide replacement of a large area of endometrium by mature squamous epithelium. Thus, a question arises: is squamous metaplasia covering a large area of endometrial surface a manifestation of endometrioid adenocarcinoma with squamous differentiation or only an independent lesion? Approximately $20 \%$ to $50 \%$ or even more of all endometrioid adenocarcinoma cases are accompanied with different degrees of squamous epithelial differentiation. Squamous differentiation is primarily man-

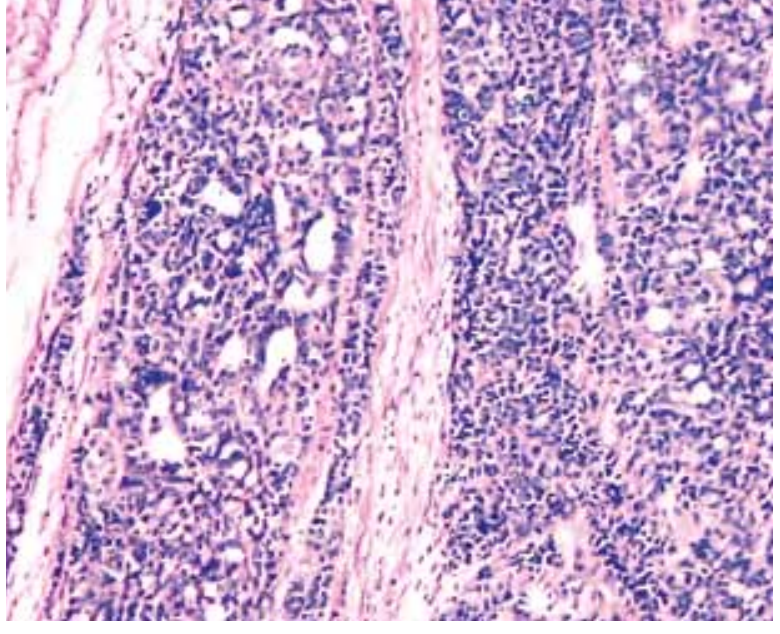

Fig. 3. Gland-like structure trends in a few tumor regions (HE; 200x)

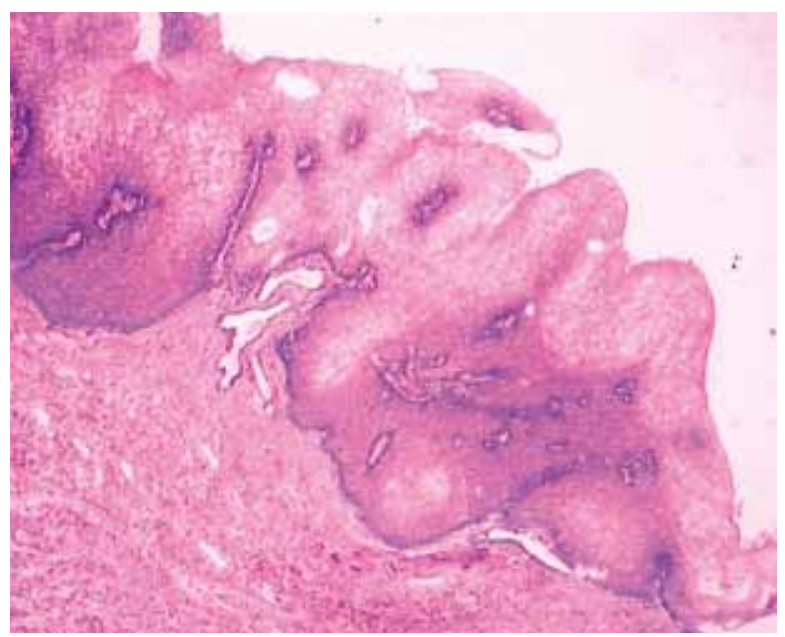

Fig 5. The endometrium surface is replaced by stratified squamous epithelium, and the endometrium glands under the squamous epithelium show atrophic changes (HE; 100x)

ifested by squamous morules, which appear multifocally in glands or in between them, indicating a close correlation with adenocarcinoma tissues. This squamous morphology was also observed in the present adenocarcinoma. The wide squamous metaplasia of the endometrium surface was manifested by mature squamous epithelium in a flattened distribution, which extensively affected most of the uterine cavity; no migration transition between the metaplasia and the inferior carcinoma was observed. These findings provide evidence that the two lesions in the present study were independent of each other as they occurred simultaneously.

Ichthyosis uteri has long been believed be a benign non-tumorous lesion; however, some scholars have also reported that squamous epithelial dysplasia [2, 5, $6,8]$ or even squamous cell carcinoma $[3,9]$ can occur. Some cases of squamous epithelial dysplasia and 
squamous cell cervical carcinoma are considered to be consequences of the direct extension of cervical lesion toward the uterine cavity, which cannot indicate the malignant potential of ichthyosis uteri. Heckeroth et al. [3] reported a patient who suffered from the development of low-grade nonspecific endometritis to ichthyosis uteri and then to uterine squamous cell carcinoma, which occurred over three years. This case suggests that ichthyosis uteri poses a risk of malignant transformation within a relatively short period of time. Similarly, Murhekar et al. [9] concluded that low-grade squamous cell carcinoma of the endometrium is associated with extensive ichthyosis uteri with dysplasia; in this case, the cervix showed moderate to severe dysplastic changes, while the right fallopian tube exhibited extensive squamous metaplasia with dysplastic changes. Furthermore, squamous cell carcinoma can develop into pre-existing ichthyosis uteri [9]. These previous reports indicate that the possibility of the malignant transformation of ichthyosis uteri cannot be completely ignored.

Ichthyosis uteri complicated by endometrial adenocarcinoma is even rarer than single ichthyosis uteri. To the best of our knowledge, only one case of ichthyosis complicated by low-grade endometrioid adenocarcinoma has been reported in the literature [5]. In contrast to that study, the present case showed a wider area of ichthyosis, which covered nearly the whole uterine cavity. In addition, noticeable squamous differentiation was observed in the endometrial adenocarcinoma in this case. The simultaneous occurrence of the two squamous forms in such noticeable degrees has not yet been reported.

The causes of ichthyosis uteri remain unknown because the disease is rare. According to reports, the causes may include chronic trauma, chronic infection, vitamin A deficiency, estrogen effect, foreign bodies, and so on. Although the clinical history of the present case failed to provide any definite cause, numerous infiltrating lymphocytes and plasmacytes around the endome- trial glands, which found under the ichthyosis squamous epithelium, suggest that the genesis of the current case was correlated with chronic inflammation.

In this study, we reported a rare case of ichthyosis uteri complicated by endometrioid adenocarcinoma with squamous differentiation. Based on previous studies and the current report, the malignant potential of ichthyosis uteri or its simultaneous occurrence with other malignant lesions cannot be completely excluded, although the lesion was considered a benign lesion. Ichthyosis uteri can only be finally diagnosed after hysterectomy. When postoperative pathology shows canceration, full materials should be drawn from the cervix uteri to exclude the possibility of its cervical source. Otherwise, clinical staging and further treatment of the disease will be greatly affected.

\section{References}

1. Marcus SL. Adenoacanthoma of the endometrium: a report of 24 cases and a review of squamous metaplasia. Am J Obstet Gynecol 1961; 81: 259-67.

2. Patton WT, Squires GV. Ichthyosis uteri. A case report. Am J Obstet Gynecol 1962; 84: 858-60.

3. Heckeroth V, Ziegler HK. Ichthyosis uteri. Case report on a rare clinical entity. Geburtshilfe Frauenheilkd 1986; 46: 248-9.

4. Kücükali T, Ertoy D, Ayhan A. Ichthyosis uteri associated with a uterine squamous papilloma. Eur J Gynaecol Oncol 1996; 17: 37-41.

5. Sherwood JB, Carlson JA, Gold MA, et al. Squamous metaplasia of the endometrium associated with HPV 6 and 11. Gynecol Oncol 1997; 66: 141-5.

6. Pins MR, Young RH, Crum CP, et al. Cervical squamous cell carcinoma in situ with intraepithelial extension to the upper genital tract and invasion of tubes and ovaries: report of a case with human papilloma virus analysis. Int J Gynecol Pathol 1997; 16: 272-8.

7. Bewtra C, Xie QM, Hunter WJ, Jurgensen W. Ichthyosis uteri: a case report and review of the literature. Arch Pathol Lab Med 2005; 129: e124-5.

8. Fadare O. Dysplastic Ichthyosis uteri-like changes of the entire endometrium associated with a squamous cell carcinoma of the uterine cervix. Diagn Pathol 2006; 1: 8.

9. Murhekar K, Majhi U, Sridevi V, Rajkumar T. Does "ichthyosis uteri” have malignant potential? A case report of squamous cell carcinoma of endometrium associated with extensive ichthyosis uteri. Diagn Pathol 2008; $3: 4$. 